\title{
Regenerative Treatment of a Cemental Tear Using Enamel Matrix Derivatives: a Ten-Year Follow-up
}

\author{
Patrick R. Schmidlin ${ }^{*}$
}

Clinic of Preventive Dentistry, Periodontology and Cariology, Center of Dental Medicine, University of Zurich, Switzerland

\begin{abstract}
A patient presented with chronic periapical periodontitis on tooth 45 . The root canal was re-treated and a wide apical perforation was closed with MTA ${ }^{\circledR}$ as an apical plug. At reevaluation six month later, the tooth presented with increased mobility, bleeding on probing and probing pocket depths of $9 \mathrm{~mm}$. Despite good periapical healing radio graphically, the tooth showed signs of localized marginal bone loss that was diagnosed as being due to a cemental fracture. The tooth was splinted, a muco-periostal flap was raised and the fragment of cementum was removed. The defect was treated in a regenerative approach, using enamel matrix derivatives (EMD). Six month after therapy, the probing pocket depths decreased to values of $\leq 3 \mathrm{~mm}$ and a defect fill was radiographically visible. The 10-year follow up showed a stable situation.

It can be concluded that the occurrence of a local delamination of the root surface may contribute to the development of plaque-induced periodontal destruction. Its removal and the regenerative conditioning of the root surface with EDTA and EMD may result in a, at least partial, resolution of the problem and regeneration of bone at the affected the site.
\end{abstract}

Keywords: Case report, cemental tear, clinical, enamel matrix proteins, follow-up, guided tissue regeneration, periodontitis.

\section{INTRODUCTION}

Structural and functional integrity of root surfaces is an essential prerequisite for the long-term preservation of periodontal health [1]. In this context, local anatomic root surface alterations may have a profound effect on gingival health, as they are considered to be plaque retentive niches. However, it is widely accepted that before periodontal disease progression can occur in the presence of pathogenic bacteria, a susceptible host and site are also required [2]. Unexpected periodontal disease progression may therefore be dependent on the simultaneous occurrence of a number of primary and secondary factors. Among the latter, enamel projections or pearls, root grooves/concavities, root fractures, and cervical root resorptions represent major tooth related factors, which may contribute to or predispose to the development of localized periodontal tissue breakdown. Cemental fractures represent a rare root surface alteration, which also fall into this group of developmental or acquired deformities and conditions and are referred to as cemental tears in the literature [3]. A recent multicenter study examined 71 teeth with cemental tears that were confirmed by direct inspection or histological examination [4]. The study showed that $56.3 \%$ of the cases were detectable on preoperative radiographs and that these teeth were indeed more likely to have periodontal bone destruction (85.9\%). Unfortunately, there is limited knowledge as to how these rare cases can effectively be treated. Case presentations still represent the predominant available source of documentation. They report

*Address correspondence to this author at the Clinic of Preventive Dentistry, Periodontology and Cariology, Center for Dental Medicine, Plattenstrasse 11, 8032 Zurich, Switzerland; Tel: +41 4463408 46;

Fax: +41 4463443 08; E-mail: patrick.schmidlin@zzm.uzh.ch on a plethora of surgical approaches and regenerative materials. Cases presenting long-term observations and outcomes of regenerative periodontal therapy are scarce.

This paper presents the regenerative periodontal treatment of an abutment tooth in a 52 year-old male, who initially presented with a symptomatic periapical lesion on a second mandibular pre-molar that had undergone previous root canal therapy. The periapical lesion was successfully retreated, but at the clinical re-evaluation 6 months after endodontic therapy, hypermobility and marginal pocket formation of $9 \mathrm{~mm}$ was observed. A radiograph revealed localized bone loss and evidence of an incomplete cervico-apical fracture of the mesial aspect, which was diagnosed as a cemental tear. The defect was micro-surgically approached; the cementum fragment was removed and the defect was treated with enamel matrix derivatives (EMD). The restorative treatment was completed with the adhesive incorporation of a fiberreinforced endodontic crown. The patient was recently reevaluated, 10 years after completion of treatment.

\section{CASE DESCRIPTION AND RESULTS}

A 64 year-old male (non-smoker, systemically healthy) presented at our clinic in 2001 with an insufficient gold bridge whose abutment teeth included the two mandibular right premolars and the mandibular right third molar. The bridge, extending from $44 / 45$ to 48 had been in service for over 25 years. His main complaint was a "bad taste". His medical history was non-contributory. His private dentist had unsuccessfully tried to treat the premolars endodontically for over 18 months. The patient had no acute symptoms, but teeth 44 and 45 had been accessed for endodontic treatment, and were left "open" intermittently for long periods. Clinical examination revealed a draining buccal sinus tract adjacent 


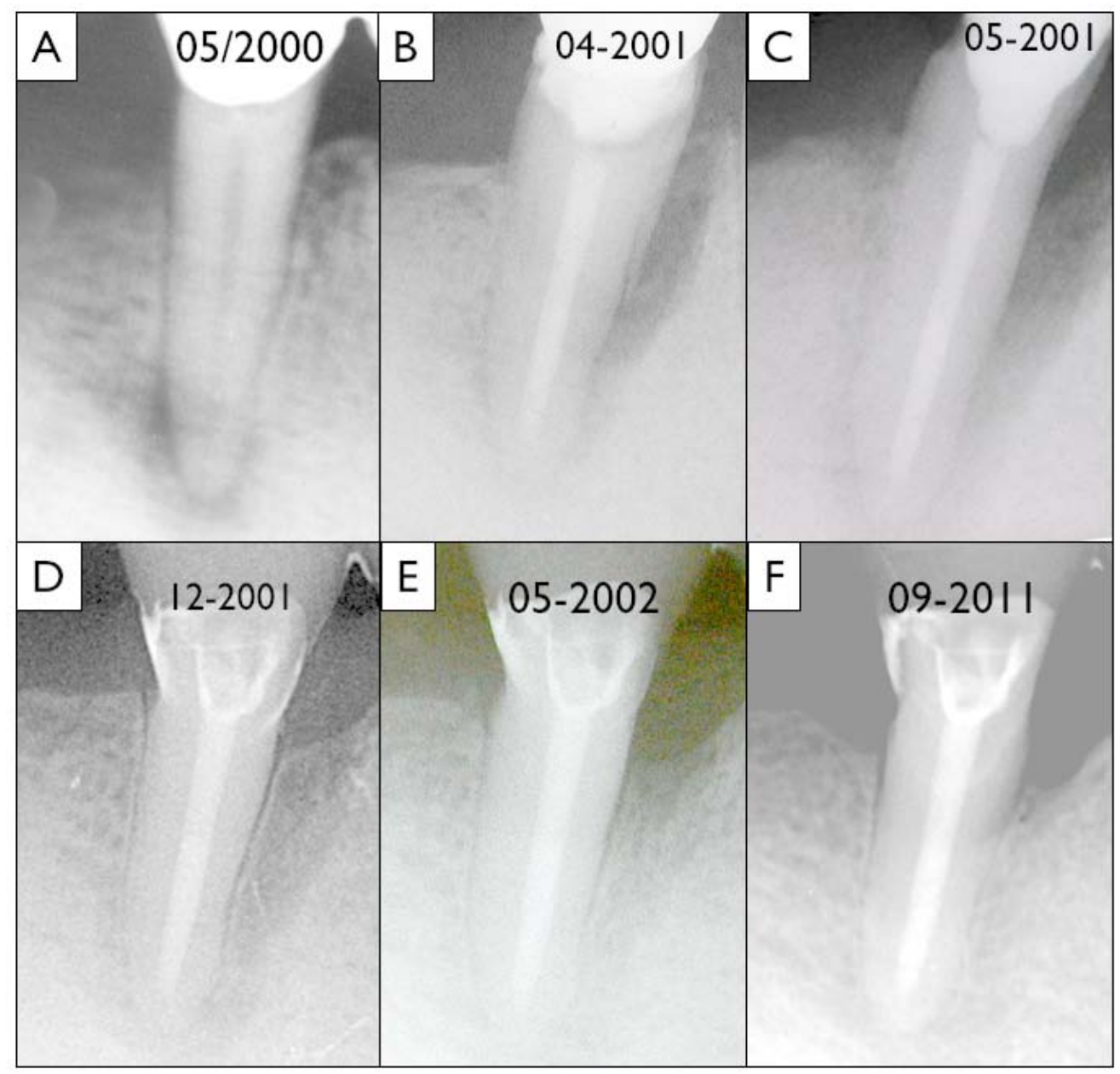

Fig. (1). Radiographic sequence of the presented case: A) baseline image of tooth 45 showing the periapical translucency in May 2000 , B) situation after the endodontic therapy and the development of a $9 \mathrm{~mm}$ pocket (April 2001), C) radiograph after periodontal surgery and ablative removal of the cemental tear (May 2001), D) immediately after restorative treatment (December 2001), E) situation one year after surgery and 6 month after oral rehabilitation (May 2002) and finally F) status after more than ten years after the surgical intervention (September 2011).

to 45 without any swelling and both premolars were nonresponsive to $\mathrm{CO}_{2}$-snow. Crown margins around the three abutments were deficient, had extensive secondary caries and the access cavities were blocked with food debris. Maximal probing depth around 44 and 45 was $4 \mathrm{~mm}$ with bleeding on probing. The preoperative radiograph showed two large apical radiolucencies with diffuse margins (Fig. 1A). A diagnosis of asymptomatic apical periodontitis was made for both teeth.

Both premolars were re-treated as follows by an endodontist (Dr. B. Lehnert): A rubber dam was applied and the root canals disinfected using $1 \% \mathrm{NaOCl}$. Visible secondary caries around the abutment margins and near the canal entrances was removed from outside. Crown Down was established (Orifice Shapers, Dentsply-Maillefer, Ballaiges, Switzerland) although the canal of 45 had already been prepared to a large diameter. Working length was determined using a size 60 file (NiTi-Flex, Dentsply-Maillefer, Ballaiges, Switzerland), which fitted loosely in the canal. Using an operating microscope, a perforation was detected at the apical foramen while some residual caries was also detected within the root canal walls. This caries was also removed from inside and sealed using a compomer according to the manufac- turer's instructions (Compoglass, Vivadent, Schaan, Lichtenstein). The canal was shaped to size 80 Lightspeed (Lightspeed, San Antonio, USA) to its working length and stepped back to size 100 . The canals were copiously irrigated using 1 $\% \mathrm{NaOCl}, 17 \%$ EDTA and $0.2 \%$ chlorhexidine alternatively. Calcium hydroxide was placed as interappointment dressing for one week and temporized using a thin layer of Cavit (Espe, Seefeld, Germany) and the canal entrance was then sealed with a glass ionomer cement (Ketac, Espe, Seefeld, Germany).

At the second appointment, one week later, the sinus tract was still visible, but without any exudate. The compomer around the crown margins was partly lost, so the margins were sealed again. At this appointment the canal was irrigated using $\mathrm{NaOCl}$, EDTA and chlorhexidine and a fresh $\mathrm{Ca}(\mathrm{OH})_{2}$ dressing was placed. The canal was not instrumented any further, due to the large diameter of the preparation.

An appointment was scheduled 18 days later, by which time the sinus tract had healed and the tooth remained asymptomatic. At this point an apical closure filling was applied using a matrix trioxide aggregate material (ProRoot, 


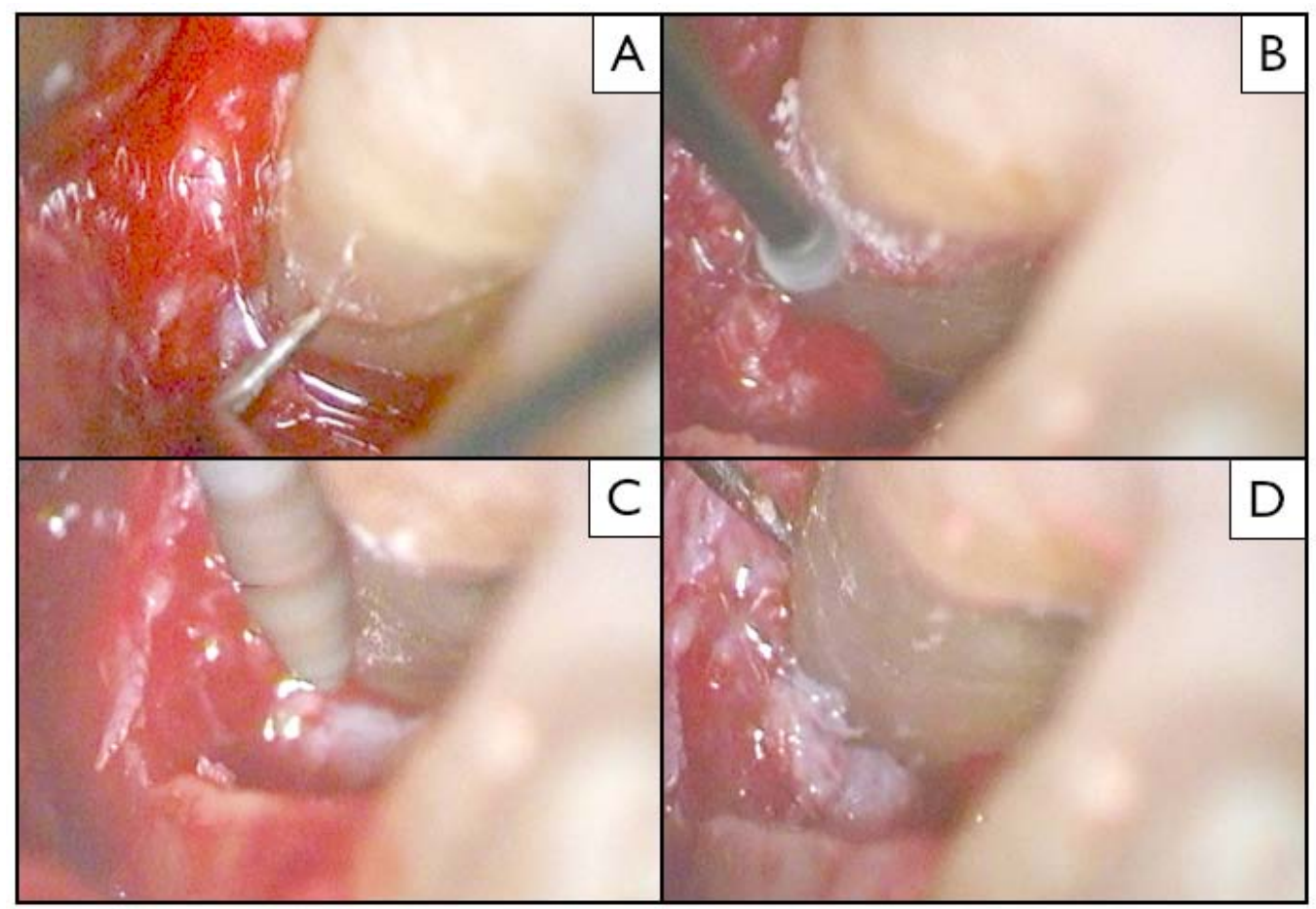

Fig. (2). Intraoperative view (snapshots of a video): A) Visible and probable fracture line, $\mathbf{B} / \mathbf{C}$ ) fragment removal using a rose head bur and a carborundum instrument, D) situation after the mechanical ablation.

Dentsply, Konstanz, Germany) to seal the apical perforation. Without any intracanal dressing, the tooth was temporised as before. Nine days later, the canal was backfilled using a thermoplastic technique (Obtura II, Spartan, Fenton, USA) and a zinc oxide-eugenol cement (Grossman's 801; Roth International, Chicago, USA). The canal entrance and the coronal 3-4 $\mathrm{mm}$ of the root canal were sealed using a dentine adhesive system (Syntac Classic, IvoclarVivadent, Schaan, Liechtenstein) and a compomer (Compglass, IvoclarVivadent, Schaan, Lichtenstein) to provide a good temporay seal until the prognosis of this tooth was clear. In order not to weaken the remaining tooth substance, posts were not considered for future treatment. The patient was referred for further comprehensive periodontal and restorative treatment.

\section{Follow-up and Occurrence of a Cemental Tear}

After completion of the endodontic therapy, the patient received routine dental prophylaxis. No special treatment was needed, as the patient did not have any pockets $\geq 3 \mathrm{~mm}$. However, at recall 6 months later, a $9 \mathrm{~mm}$ pocket was detected mesially and lingually on tooth 45 , with excessive bleeding on probing. A diagnostic radiograph showed marginal bone loss and a significant, localized tear-like fracture of the root (Fig. 1B). The original apical periodontitis showed clear signs of advanced healing at this time.

The diagnosed cemental tear was considered to be the primary etiological factor. As a cause-related therapy, we proposed surgical removal of the fragment and application of EMD, in a regenerative approach. Before the surgical intervention, the defective bridge was removed and both abutment premolars displayed an increased mobility of grade 3 . They were then splinted together using composite and a fibrepontic for reinforcement as follows: After rubberdam placement, the provisional restorative material was removed and an adhesive system was applied on the coronal dentin according to the manufacturer's instructions (Syntac Classic and Tetric, Ivoclar Vivadent). A fibrepontic (Vectris, IvoclarVivadent) was adapted from tooth 45 to 43 for mechanical reinforcement in order to splint the mobile teeth for further therapy.

\section{Periodontal Surgery}

Under local anesthesia, a full thickness muco-periostal flap was raised buccally and lingually after an intrasulcular incision. No vertical incisions were made to avoid compromising the blood supply to the flaps. Granulation tissue was removed from the defects and the fracture line was clearly visible (Fig. 2A). The fragment could not be mobilized and removed in toto for histological evaluation. Therefore, the fragment was removed using burs under constant watercooling (Figs. 1C, 2B-D). The surface was debrided using an ultrasonic scaler (Mini Piezon, EMS, Nyon, Switzerland) and the remaining surface was smoothed using a series of diamond-coated burs (Intensiv SA, Viganello, Switzerland). After careful bleeding control, the root surface was treated with 24\% EDTA gel (Biora AB, Malmö, Sweden) for 2 minutes, the area was rinsed with sterile saline. Emdogain ${ }^{\circledR}$ gel(Biora AB, Malmö, Sweden; currently: Straumann, Basel, Switzerland) was applied to the defects using a sterile syringe. Pre-sutured flaps were fixed using a vertical mattress technique and the patient was instructed to rinse with $0.2 \%$ chlorhexidine (Hibitane, Kantonsapotheke, Zürich, Switzerland) twice daily for two weeks. The patient was given oral analgesics (Ponstan, Pfizer, Zurich, Switzerland) for use when required. No antibiotics were prescribed. Sutures were removed 2 weeks after surgery. Oral hygiene was comprised of tooth brushing, but no use of interdental 


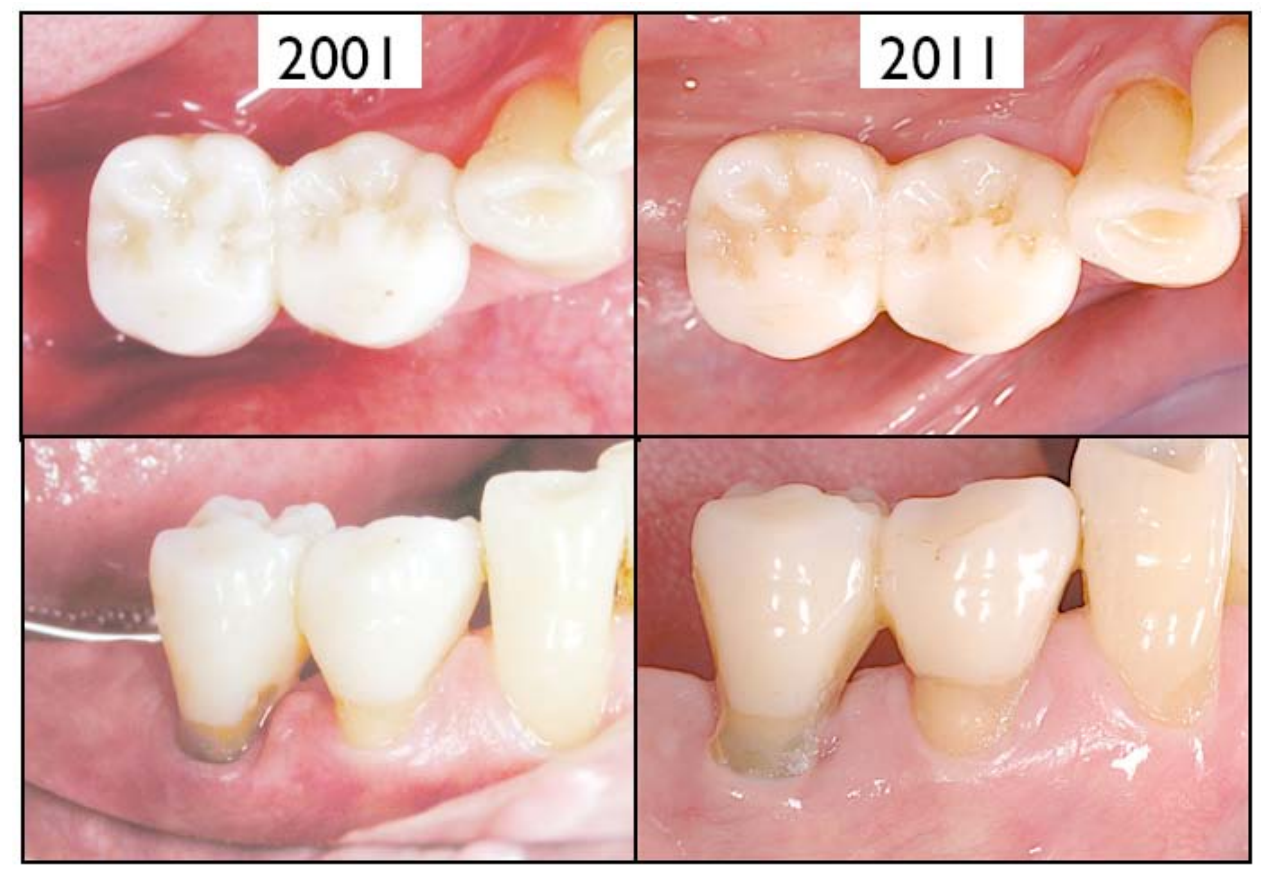

Fig. (3). Clinical situation after restoration (year 2001, left) and after 10 years (right). Some generalized recession had developed. Some plaque is visible. Otherwise the situation is stable. No probing pocket depths $>4 \mathrm{~mm}$ were recorded.

brushes, for the first 3 months. The patient was recalled at 3 and 6 months. At the six-month re-evaluation, no pockets $\geq 3$ $\mathrm{mm}$ and no bleeding on probing were detected. Treatment was completed with the adhesive placement of an indirect fiber-reinforced endocrown without post but a central retention cavity inside the pulp chamber (Targis-Vectris, IvoclarVivadent, Schaan, Liechtenstein). One year after surgery and 6 month after restorative treatment, there were still no clinical signs of inflammation or pocket formation. The radiograph showed evidence of good endodontic and periodontal healing with signs of the formation of a periodontal ligament (Fig. 1D, E). The patient then left our clinic and was referred back to a private dentist. Ten years after surgery, we were able to contact the patient and re-examine the situation.

A radiograph showed a stable bone levels, with only delicate remodeling and bone loss at the affected site. Bone density and the periodontal ligament were still well preserved and consolidated (Fig. 1F). Clinically, maximum probing depths were $4 \mathrm{~mm}$ with bleeding on probing at the mesial and distal site of tooth 45 and 44 . Tooth mobility was not increased (grade 1). Oral hygiene was not adequate, especially interdentally, where considerable amounts of plaque were visible (Fig. 3). The patient admitted that he did not attend regular recalls during the last five years, due to health problems and other personal reasons. Given this fact, the stable clinical and radiographic results were impressive.

\section{DISCUSSION}

The diagnosis of a cemental tear is always difficult. In this particular case, the periodontal problems, which had arisen suddenly after successful endodontic re-treatment could be related to the radiographically distinguishable disintegration of the root surface, resulting in a cemental tear. Adequate knowledge of the predisposing factors leading to defect formation are a prerequisite to cause-related therapy.
According to Lin and co-workers [4], univariate analysis of predisposing factors found that teeth with cemental tears occurred more commonly in men $(77.5 \%)$ and patients older than 60 years of age (73.2\%), which was in accordance with the present case. However, the latter study found that maxillary and mandibular incisors (76.1\%) were most frequently affected. In the present case, a premolar was involved. Further analysis of clinical characteristics showed that teeth with cemental tears were prone to have abscess formation (66.2\%), and a deep periodontal pocket $>6 \mathrm{~mm}(73.2 \%)$. In this case, a deep pocket was also found but there was no abscess. Lin and co-workers also showed that affected teeth were more likely to have a positive vitality test $(65.3 \%)$, healthy antagonist teeth (84.3\%), and moderate to severe attrition $(77.9 \%)$. In our patient, the alteration occurred after root canal therapy, but in accordance with the earlier findings, the opposing dentition was healthy and showed some signs of bruxism in terms of wear facets. We can also speculate that the insufficient superstructure and overloading may have contributed to the development of the cemental tear.

Whereas diagnosis and evaluation of the etio-pathogenic factors is one hurdle, the appropriate and best treatment solution is certainly another. Due to the small number of cases, only case reports and no interventional or controlled studies evaluating and comparing different treatment modalities are available so far. The existing body of literature, however, strongly suggests that a removal of the defective tooth part is prerequisite as it represents a plaque retentive and causative factor. The surgical management of the bony defect greatly depends on the defect type, as well as patient characteristics and compliance. Whereas conventional surgical periodontal therapy leads to a recovery of the periodontal tissues by reparative wound healing [5], materials and techniques are now available which stimulate regeneration of the original tissues, i.e. guided tissue regeneration (GTR) [6,7]. There is 
evidence of effectiveness, especially when dealing with infrabony and furcation defects [8]. In the 1990's, enamel matrix derivates (EMD; Emdogain ${ }^{\circledR}$, Biora, Malmö, Sweden and later Straumann, Basel, Switzerland) became commercially available. They allow for regenerative procedures without the necessity of applying membranes and/or filler materials [9]. The use of EMD has been first demonstrated in animal [10] and later in human studies [11]. Several systematic articles have systematically compared this approach to classical flap procedures and open debridement and have evaluated the additional benefit in the clinical outcomes [8, 12-14]. These reviews underline that EMD may exhibit a measurable positive clinical effect in combination with surgical treatment of periodontally diseased teeth when treating infrabony defects and furcations, provided that patients' compliance is adequate and correct indications are pursued, including: careful assessment of defect depth, number of residual bony walls, pocket depth, and the degree of hypermobility. According to a literature review by Kasaj and coworkers, seven single-case reports using bone graft substitutes with or without GTR were identified [1, 15-20], but only one case reporting that the use of EMD was available [21]. The latter revealed a successful outcome using EMD after an observation period of 1 year. The observation period of the other case presentations ranged from 4 months to 4 years.

Within the limitation of this single case, it can be summarized according to Lin and co-workers that clinical signs such as tissue swelling, narrow deep pocket formation, the presence of a radiopaque fragment on the root surface and the related periodontal/periapical bone destruction are major features of teeth with cemental tears. The removal of the detached fragment and/or the mechanical root surface modification to achieve a smooth and unaltered root surface is a prerequisite for any surgical treatment. The latter can be accomplished by several different approaches. Guided tissue regeneration may lead to bone gain and a prolonged survival rate.

\section{CONFLICT OF INTEREST}

The author confirms that this article content has no conflicts of interest.

\section{ACKNOWLEDGEMENT}

Declared none.

\section{REFERENCES}

[1] Haney JM, Leknes KN, Lie T, Selvig KA, Wikesjo UM. Cemental tear related to rapid periodontal breakdown: a case report. J Periodontol 1992; 63: 220-4.
[2] Leknes KN. The influence of anatomic and iatrogenic root surface characteristics on bacterial colonization and periodontal destruction: a review. J Periodontol 1997; 68: 507-16.

[3] Armitage GC. Development of a classification system for periodontal diseases and conditions. Ann Periodontol 1999; 4: 1-6.

[4] Lin HJ, Chan CP, Yang CY, et al. Cemental tear: clinical characteristics and its predisposing factors. J Endod 2011; 37: 611-8.

[5] Caton JG, Zander HA. The attachment between tooth and gingival tissues after periodic root planing and soft tissue curettage. J Periodontol 1979; 50: 462-6.

[6] Gottlow J, Nyman S, Lindhe J, Karring T, Wennström J. New attachment formation in the human periodontium by guided tissue regeneration. Case reports. J Clin Periodontol 1986;13: 604-16.

[7] Pontoriero R, Nyman S, Lindhe J, Rosenberg E, Sanavi F. Guided tissue regeneration in the treatment of furcation defects in man. $J$ Clin Periodontol 1987;14: 618-20.

[8] Cortellini P, Tonetti MS. Focus on intrabony defects: guided tissue regeneration. Periodontol 2000 2000; 22: 104-32.

[9] Sculean A, Schwarz F, Becker J, Brecx M. The application of an enamel matrix protein derivative (Emdogain) in regenerative periodontal therapy: a review. Med Princ Pract 2007;16: 167-80.

[10] Hammarstrom L. Enamel matrix, cementum development and regeneration. J Clin Periodontol 1997; 24: 658-68.

[11] Yukna RA, Mellonig JT. Histologic evaluation of periodontal healing in humans following regenerative therapy with enamel matrix derivative. a 10-case series. J Periodontol 2000; 71: 752-9.

[12] Esposito M, Grusovin MG, Coulthard P, Worthington HV. Enamel matrix derivative (Emdogain) for periodontal tissue regeneration in intrabony defects. Cochrane Database Syst Rev 2005; (4) CD003875.

[13] Needleman IG, Worthington HV, Giedrys-Leeper E, Tucker RJ. Guided tissue regeneration for periodontal infra-bony defects. Cochrane Database Syst Rev 2006; (2): CD001724.

[14] Tu YK, Tugnait A, Clerehugh V. Is there a temporal trend in the reported treatment efficacy of periodontal regeneration? a metaanalysis of randomized-controlled trials. J Clin Periodontol 2008; 35: $139-46$

[15] Muller HP. Cemental tear treated with guided tissue regeneration: a case report 3 years after initial treatment. Quintessence Int 1999; 30: 111-5.

[16] Harrel SK, Wright JM. Treatment of periodontal destruction associated with a cemental tear using minimally invasive surgery. J Periodontol 2000; 71: 1761-6.

[17] Camargo PM, Pirih FQ, Wolinsky LE, Lekovic V, Kamrath H, White SN. Clinical repair of an osseous defect associated with a cemental tear: a case report. Int J Periodontics Restorative Dent 2003; 23: 79-85.

[18] Marquam BJ. Atypical localized deep pocket due to a cemental tear: case report. J Contemp Dent Pract 2003; 4: 52-64.

[19] Lyons CT, Peacock ME, Cuenin MF, Swiec GD, Dickey DJ. Severe localized periodontal destruction associated with cervical cemental separation. Gen Dent 2005; 53: 212-4.

[20] Tulkki MJ, Baisden MK, McClanahan SB. Cemental tear: a case report of a rare root fracture. J Endod 2006; 32: 1005-7.

[21] Kasaj A, Gortan-Kasaj A, Briseno-Marroquin B, Willershausen B. Treatment of severe localized periodontal destruction associated with a cemental tear: a case report and review of the literature. Gen Dent 2009; 57: e 5-9.

(c) Patrick R. Schmidlin; Licensee Bentham Open.

This is an open access article licensed under the terms of the Creative Commons Attribution Non-Commercial License (http://creativecommons.org/licenses/by-nc/3.0/) which permits unrestricted, non-commercial use, distribution and reproduction in any medium, provided the work is properly cited. 